\title{
Cuerpo extraño pulmonar: resección por videotoracoscopia. Caso clínico*
}

\author{
Drs. JORGE SALGUERO A. ${ }^{1}$, ANDRÉS MARAMBIO G. ${ }^{1}$, \\ FERNANDA BAEZA G. ${ }^{2}$, Al. JUAN P. MARAMBIO G. ${ }^{3}$ \\ Departamento de Cirugía. \\ 2 Departamento de Anestesia. \\ 3 Alumno Facultad de Medicina. \\ Hospital Clínico de la Universidad de Chile, Santiago, Chile.
}

\begin{abstract}
Inhaled foreign body: a video-thoracoscopic resection. A case report

We report an 18 years old male who reported a history of 2 years of recurrent episodes of hemoptysis with no other symptoms. A chest X-ray and chest CT scan, showed an image suggestive of a metallic foreign body in the posterior segments of the right lower lobe. Since fibro-bronchoscopy failed to extract it, a video-thoracoscopy with wedge resection of the affected lung parenchyma was performed, finding a capsular lesion of inflammatory tissue which contained a map pin in its interior. The clinical outcome was favorable, with a chest X-ray showing proper lung re-expansion. The patient was discharged 48 hours after surgery.
\end{abstract}

Key words: Hemoptysis, lung foreign body, video-thoracoscopy.

\section{Resumen}

Se reporta el caso de un paciente hombre de 18 años con un cuerpo extraño pulmonar de presentación tardía, que consultó por un cuadro de 2 años de evolución de episodios de hemoptisis escasa y recurrente, sin otros síntomas asociados. Se realizó estudio con radiografía y TAC de tórax evidenciándose una imagen sugerente de un cuerpo extraño metálico en los segmentos posteriores del lóbulo inferior derecho. Dado que la fibrobroncoscopia resultó frustra se decidió realizar una videotoracoscopia con resección en cuña del parénquima pulmonar afectado, encontrándose una lesión capsular de tejido inflamatorio que en su interior contenía un chinche. La evolución clínica fue favorable, con radiografía de tórax de control con adecuada reexpansión pulmonar y dándose de alta a las $48 \mathrm{hrs}$ postoperatorias.

Palabras clave: Hemoptisis, cuerpo extraño pulmonar, videotoracoscopia.

\footnotetext{
*Recibido el 22 de Diciembre de 2008 y aceptado para publicación el 24 de Febrero de 2009.

Correspondencia: Dr. Jorge Salguero A.

Santos Dumont 999, Santiago, Chile. Fax: 56-2-7370844

E-mail: jorsal@mi.cl
} 


\section{Introducción}

La aspiración de cuerpo extraño (CE) es una patología que suele verse en la población pediátrica, principalmente entre el primer y tercer año de vida ${ }^{1-3}$, período en el cual el niño experimenta con todo lo que encuentra a su alrededor, llevándose a la boca diversos objetos y pudiendo alguno de estos migrar hacia la vía aérea. Aunque habitualmente es un cuadro súbito, a veces puede pasar desapercibido llevando a retraso en el diagnóstico y pesquisándose en edades más avanzadas. La forma de presentación es variable dependiendo del tamaño, ubicación del CE y momento del diagnóstico ${ }^{4}$. Así el cuadro clínico puede ir desde una obstrucción severa de la vía aérea que compromete la vida, hasta episodios de tos, sibilancias, hemoptisis, infecciones recurrentes, etc ${ }^{1,3,5,6}$. De esta forma el estudio y tratamiento dependerá del tipo de presentación y gravedad del cuadro. Aunque habitualmente el tratamiento es endoscópico mediante una fibrobroncoscopia, la cual ha demostrado ser un método efectivo y seguro, en ocasiones y debido a la ubicación del CE o de sus características físicas, el tratamiento requerirá de su extracción quirúrgica, ya sea por videotoracoscopia (VTC) o toracotomía?. A continuación reportamos el caso de un paciente con un cuerpo extraño pulmonar de presentación tardía en el que se realiza una VTC para su extracción.

\section{Caso clínico}

Paciente hombre de 18 años, sano, que consulta por un cuadro de dos años de evolución, de episodios autolimitados de hemoptisis escasa, recurrentes y relacionados en ocasiones a la actividad física. Sin antecedentes de alguna otra sintomatología respiratoria asociada, fiebre, baja de peso, tabaquismo o hallazgos positivos al examen físico. Debido a esto se le solicitaron exámenes para descartar etiología infecciosa y una radiografía de tórax PA-Lateral la cual evidenciaba una imagen radiopaca, lineal y con densidad metálica sobre proyectada en segmentos basales posteriores del lóbulo inferior derecho (Figuras 1 y 2). Se complementa el estudio con una tomografía axial computada de tórax en la cual se observa en el segmento basal posterior derecho estructuras bronquiales dilatadas asociadas a aumento del intersticio peribroncovascular y en la que destaca una imagen espontáneamente densa endoluminal que podría corresponder a un CE metálico (Figura 3). Por estos hallazgos se realiza una fibrobroncoscopia flexible para la ubicación y posterior extracción del $\mathrm{CE}$, sin embargo, el endos-

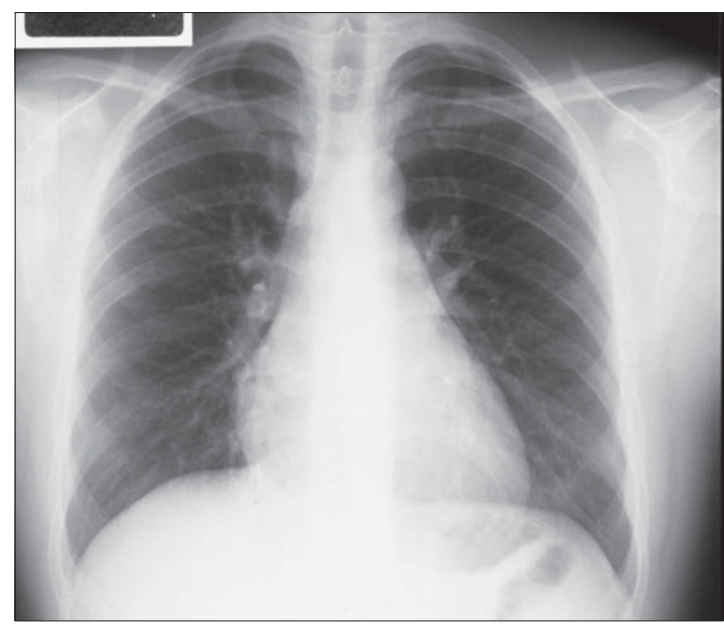

Figura 1. Radiografía de tórax PA con CE metálico lineal en segmento posterior del lóbulo inferior derecho.

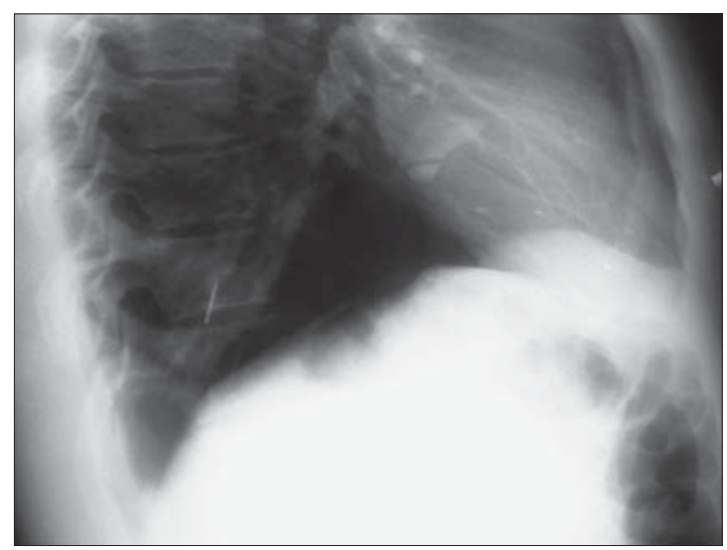

Figura 2. Proyección lateral.

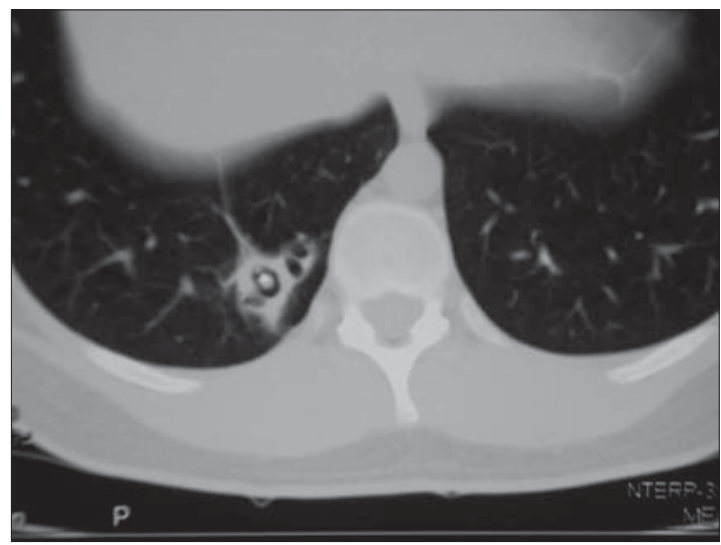

Figura 3. TAC de tórax que muestra estructuras bronquiales dilatadas asociadas a tejido fibroso con CE metálico en su interior. 


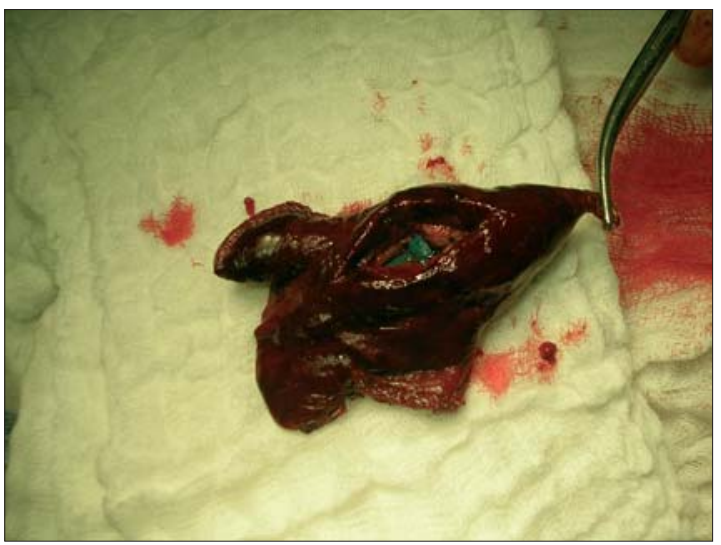

Figura 4. Cuña de parénquima pulmonar de aspecto solevantado e indurado. Al realizar corte con el bisturí se observa un CE plástico.

copista no logró acceder hasta el objeto. Debido a esto se solicita evaluación por el equipo de cirugía de tórax decidiéndose su extracción quirúrgica por VTC. En pabellón y mediante tres trocares se ingresa a la cavidad torácica evidenciándose un parénquima pulmonar de aspecto sano, sin adherencias. Con el pulmón en deflación se observa una región circular, solevantada e indurada, en la región posterior de la superficie diafragmática del lóbulo inferior derecho. Se realiza una resección en cuña de toda la zona de $5 \times 4 \times 2 \mathrm{~cm}$, sin incidentes. Al extraer la pieza operatoria, esta se escinde encontrándose un "chinche" en su espesor, rodeado por una cápsula de tejido inflamatorio (Figuras 4 y 5). El paciente evoluciona satisfactoriamente y la radiografía de tórax PA a las $7 \mathrm{~h}$ post cirugía muestra una adecuada reexpansión pulmonar (Figura 6). Se le retira el drenaje pleural a las $48 \mathrm{hrs}$ siendo dado de alta ese mismo día. La biopsia definitiva reveló la presencia de bronquiectasias con mucosa erosionada e infiltrado inflamatorio mononuclear asociado a fibrosis y hemorragia alveolar focal.

La madre manifiesta posteriormente que el paciente había eliminado un cuerpo extraño similar por las heces a los 6 años de edad.

\section{Discusión}

Sobre este caso hay varios aspectos interesantes que comentar. En primer lugar hacer mención a la edad del paciente al momento de la presentación del cuadro clínico. Como ya se dijo, esta es una patología de mayor incidencia en los primeros años de la vida, aunque se han reportado latencias de

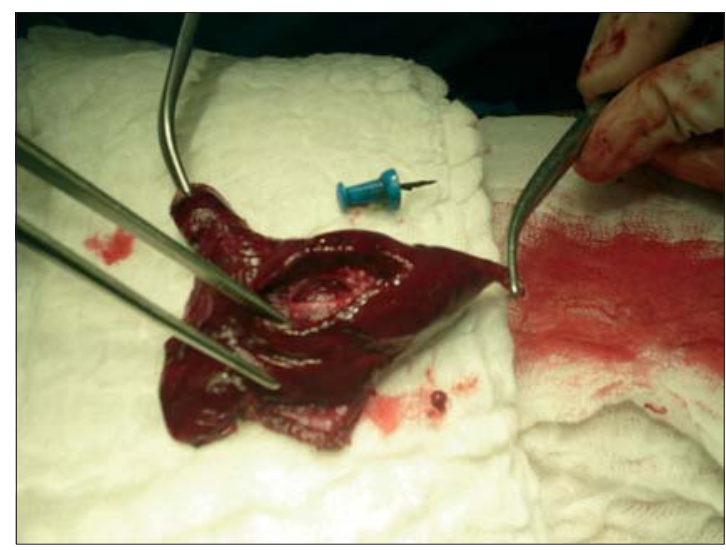

Figura 5. Se extrae completamente el CE evidenciándose que corresponde a un chinche.

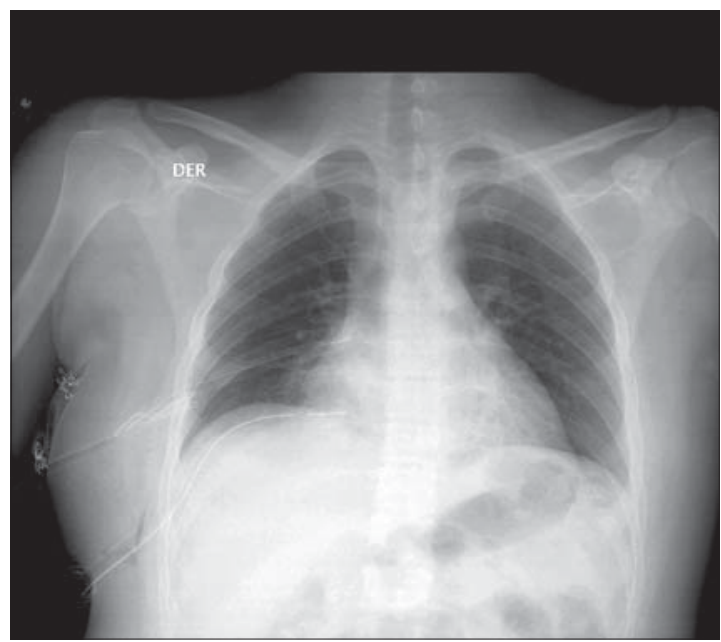

Figura 6. Radiografía de tórax portátil de control postoperatoria con dos tubos de drenaje pleural in situ.

hasta 10 años $^{4}$, en casos de aspiración no presenciados por adultos, con pocos síntomas al inicio y que posteriormente son tratados en ocasiones por infecciones recurrentes. Este paciente inicia la sintomatología a la edad de 16 años quedando la aspiración de $\mathrm{CE}$ como un diagnóstico poco probable; del mismo modo la hemoptisis es una forma infrecuente de presentación clínica ${ }^{6}$. Es así como en pacientes jóvenes aún se debe considerar esta etiología frente a cuadros pulmonares de presentación atípica o que no responden bien al tratamiento. Cabe mencionar que se ha visto que en la medida que el diagnóstico se retrasa, este CE se empie- 
za a rodear por una reacción inflamatoria que lo envuelve, haciendo más difícil su posterior extracción; y que también a mayor permanencia del CE en la vía aérea, la posibilidad de que se complique (bronquiectasias, enfisema, neumonías a repetición, neumotórax, neumomediastino, enfisema subcutáneo) aumenta en forma progresiva ${ }^{4}$. En este caso a pesar de la demora en el diagnóstico y tratamiento definitivo, el paciente no había presentado otras complicaciones clínicas aparte de los episodios de hemoptisis.

En cuanto a la etiología, los estudios sitúan a los CE orgánicos como los elementos más frecuentemente encontrados. En especial destaca la aspiración de maní ${ }^{1-5,8}$ como primera causa en todos los estudios, seguido de otros elementos orgánicos y habitualmente en los segmentos inferiores del lóbulo inferior derecho $0^{5,8-10}$. Debido a esto la radiografía inicial suele ser negativa requiriendo una alta sospecha clínica para llegar al diagnóstico. En este caso en particular nos encontramos con un chinche, de mayor tamaño que un maní y que, como se aprecia en las radiografías iniciales, se encuentra con su superficie roma hacia distal permitiendo su migración por el árbol bronquial hasta una posición inalcanzable para la fibrobroncoscopia. Además la forma, disposición, tamaño y reacción inflamatoria que presentaba este CE hubiesen impedido su extracción por esta vía. Es por esto que se realiza una VTC, técnica que se caracteriza por tener una baja incidencia de complicaciones pulmonares postoperatorias, asociado a menor dolor y una recuperación y alta más precoz. Otros casos reportados de CE en vía aérea también han sido extraídos mediante cirugía, principalmente mediante toracotomía ${ }^{6,7,9}$. En este paciente, la VTC asociada a un $\mathrm{CE}$ periférico, nos permitió realizar una resección en cuña localizada del parénquima pulmonar comprometido, sin requerir de lobectomía o segmentectomía, como se ha reportado en otros casos de CE complicados, de vías aéreas más centrales. No conocemos reportes nacionales de extracción de CE por VTC, por lo que nos parece de interés comunicar a la comunidad quirúrgica este caso, destacando que de lo mencionado en nuestro reporte se puede deducir que el paciente tuvo alojado el CE por 12 años.

\section{Referencias}

1. Baharloo F, Veyckemans F, Francis C, Biettlot MP, Rodenstein DO. Traqueobronchial Foreign Bodies: Presentation and management in Children and Adults. Chest. 1999; 115: 1357-1362.

2. Hui H, Na L, Zhijun CJ, Fugao ZG, Yan S, Niankai $\mathrm{ZK}$, et al. Therapeutic experience from 1428 patients with pediatric tracheobronchial foreign body. J Pediatr Surg 2008; 43: 718-721.

3. Tan HK, Brown K, McGill T, Kenna MA, Lund DP, Healy GB. Airway foreign bodies (FB): a 10-year review. Int J Pediatr Otorhinolaryngol 2000; 56: 9199.

4. Sersar SI, Rizk WH, Bilal M, El Diasty MM, Eltantawy TA, Abdelhakam BB, et al. Inhaled foreign bodies: presentation, management and value of history and plain chest radiography in delayed presentation. Otolaryngol Head Neck Surg 2006; 134: 92-99.

5. Swanson KL, Prakash UB, Midthun DE, Edell ES, Utz JP, McDougall JC, et al. Flexible bronchoscopic management of airway foreign bodies in children. Chest 2002; 121: 1695-1700.

6. Zúñiga S, Mañana M, Monge M, García C, González S, Iturriaga L, y cols. Cuerpo Extraño Vegetal en la vía aérea: una causa poco frecuente de hemoptisis en la niñez. Caso clínico. Rev Méd Chile 2000; 128: 323-329.

7. Dave N, Oak SN. Metallic foreign body in the lung: a case report. J Pediatr Surg 2007; 42: 1282-1283.

8. Tang FL, Chen MZ, Du ZL, Zou CC, Zhao YZ. Fibrobronchoscopic treatment of foreign body aspiration in children: an experience of 5 years in Hangzhou City, China J Pediatr Surg 2006; 41: 1-5.

9. Ülkü R, Onen A, Onat S, Özçelik C. The value of open surgical approaches for aspirated pen caps. J Pediatr Surg 2005; 40: 1780-1783.

10. Moreno A, Bordagaray P, Aravena M, Valdebenito G, Stevens A. Cuerpos extraños en la Vía Aérea Inferior. Rev Chil Pediatr 1983; 54: 91-95. 